\title{
Enhanced Ti I spectral intensity using NELIBS technique
}

\author{
Original \\ Ahmed H. El Farash ${ }^{1}$, Ashraf M. El Sherbini ${ }^{2}$, Osama Mohamed Said Helal ${ }^{1}$, \\ Article \\ Ashraf F. El-Sherif ${ }^{1}$ \\ ${ }^{I}$ Department of Engineering Physics, Military Technical College, ${ }^{2}$ Laboratory of Laser \\ and New Materials, Faculty of Science, Cairo University, 12613, Giza, Egypt.
}

\section{Keywords:}

Enhancement, nano-gold, nanoparticles, NELIBS, plasma.

\section{Corresponding Author:}

Ahmed H. El Farash, Department of Engineering Physics, Military Techincal College, Tel: $\quad+201010724698$. Email: a.h.elfarash@mtc.edu.eg.

\section{Abstract}

Enhanced emission from nano coated titanium target over than that from normal solid one was measured. The plasma was induced from both targets by the interaction of Nd: YAG laser source at a wavelength of $1064 \mathrm{~nm}$. The enhanced signal emission was monitored using the Ti I- spectral lines at 453.32 and $498.17 \mathrm{~nm}$. Another experiment was conducted after addition of a thin layer of $20 \mathrm{~nm}$ gold nanoparticles to the surface of bulk titanium. Enhancement factors were found up to twenty folds. The plasma-enhanced emission was attributed to the strong reduction in the plasma ignition threshold by a factor of 40 after adding the layer ofgold nanoparticles. Modeling to explain the origin of the strong reduction to the ignition threshold and enhanced emission is in advance which recommended that there a strong reduction of the thermal diffusion length of the bulk material to the limits of nanomaterial diameter size.

\section{INTRODUCTION}

Laser-induced breakdown spectroscopy technique (LIBS) is classified among the most important techniques in the spectrochemical analysis ${ }^{[1]}$. At first, the analyzed material is converted into a plasma state upon irradiation with high peak power pulsed lasers ${ }^{[2]}$. It was assumed that the light emitted from this plasma is sufficiently influenced by the elemental structure of the sample as well as the concentration of the different ingredients ${ }^{[3]}$. A wealth of information can be gained upon analysis of the light emitted from this pulsed plasma ${ }^{[1,2]}$ and therefore it was recommended in many of spectrochemical analytical applications $^{[4-6]}$, e.g. in the field of biochemistry ${ }^{[7]}$ space exploration $^{[8]}$, environmental, forensic sciences $^{[9]}$, as well as industrial applications ${ }^{[10]}$ and archeology applications ${ }^{[11]}$. This technique contains many advantages over other conventional analytical techniques because of its non-destructive nature, a high degree of reliability, realtime results and almost there is no sample preparation ${ }^{[12]}$. However, this technique is relatively poor in detecting the very rarefied elemental concentrations $(\sim 10 \mathrm{ppm})^{[13]}$ of trace elements inside the examined sample because the intensity of the prominent lines decreases to values that are comparable to the noise level ${ }^{[14]}$. The limit of detection (LOD) was taken as the main parameter to determine the lower limit of detection of the rarefied concentrations ${ }^{[15]}$. This LOD depends on two main factors, the signal strength (height) and the background radiation. Sincere efforts were provided to overcome this problem utilizing different techniques which include the use of double-pulse laser technique ${ }^{[1,16,17]}$, the use of ultra-short laser pulses to excite plasma ${ }^{[18,19]}$ or a blended technique ${ }^{[20,21]}$. But these techniques depend on raising the laser abilities that require a new laser source that needs to change the experimental set up as well as increasing the overall cost.

Modified LIBS-Technique was introduced as Nanoparticle-Enhanced laser-induced breakdown spectroscopy (NELIBS) in order to enhance the signal intensity emerged from the rarefied concentrations. It takes advantage of the daily progress of nanotechnology utilizing its unique physical properties. This leads to improving the detection sensitivity to sub-ppm values $^{[22,23]}$. It was noticed that this additive greatly enhances the optical signal arising from the bulk material ${ }^{[24]}$.

One possible explanation based on considering excitation of the surface plasmon ofNano-particles which leads to the early ignition of plasma from the surface of metals $^{[23]}$. Another acceptable explanation based on the strong reduction of the plasma ignition threshold ${ }^{[24]}$. This decrease in the ignition threshold was noticed to depend on the size and the type of the used nanoparticle material as well as the exciting laser wavelength in an inverse square way $^{[14]}$

In this article, we shall demonstrate the enhancement of plasma emissions from titanium nanoparticles over bulky one in LIBS. Plasma emission was monitoredvia following the Ti I-lines at wavelengths of 453.32 and $498.17 \mathrm{~nm}$, before and after the addition of a thin layer of Nano-gold particles to the surface of bulk titanium target. 


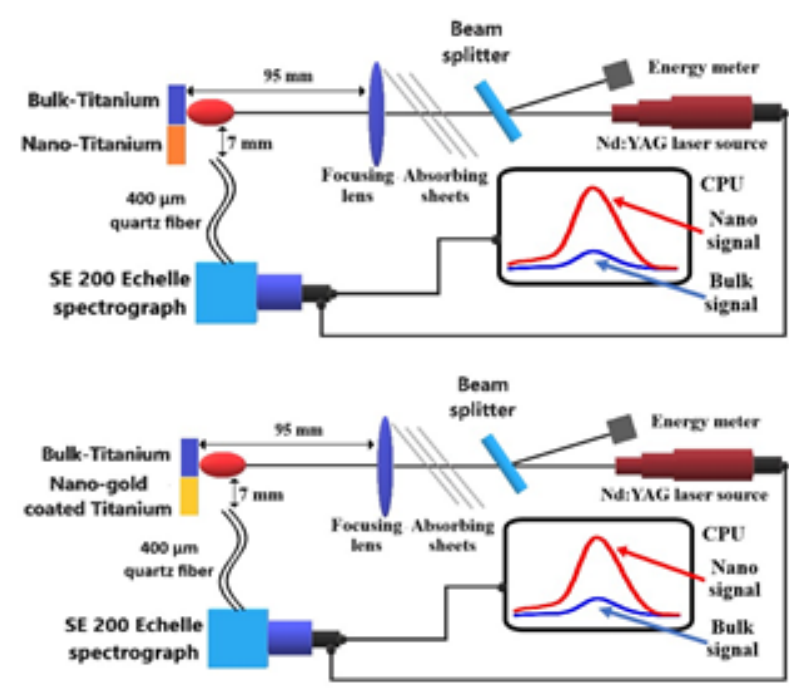

Fig. 1: Experimental setup. (a) Nano Titanium, (b) Nano-gold coated titanium.

\section{EXPERIMENTAL WORK}

The utilized two experimental setups are shown in Fig. 1 are slightly different in the target as (a) the target surface is divided between bulk and nanoparticles titanium however, (b) the target's two sides are bulk and nanogold deposited titanium. It comprises Nd-YAG laser (type Quantel-Brilliant B) working at the fundamental wavelength of $1064 \mathrm{~nm}$ with output maximum laser energy of $370 \pm 5 \mathrm{~mJ}$ per pulse within $5 \mathrm{~ns}$ duration. The laser spot size was adjusted at the target surface at avoid breakdown the surrounding air by changing the distance from focusing lens to target $98 \pm 1 \mathrm{~mm}$ and was measured at the position of the target using a special thermal paper (supplied with Quantel®) found almost of circular shape of radius $0.7 \pm 0.05 \mathrm{~mm}$ at the laser wavelength $1064 \mathrm{~nm}$. This routine granted that the plasma emission is originated from the target rather than ambient air. The light from the plasmas was collected using $400 \mu \mathrm{m}$ diameter optical fiber $(\mathrm{NA}=0.22)$ to the entrance hole of the SE200- Echelle type spectrograph (optical resolution of $0.02 \mathrm{~nm}$ per pixel with an instrumental bandwidth of $0.2 \mathrm{~nm}$ on the average).

The emitted light from the plasma was spatially resolved and spectra were acquired using a fast response ICCD camera (Andor- iStar DH734-18F) and the data control was carried using KestrelSpec $\AA 3.96$ software with a resolution of $0.02 \mathrm{~nm}$ per pixel of size $196 \mu \mathrm{m} 2$. The optical fiber was positioned at a distance of $7 \pm 1 \mathrm{~mm}$ from the laser-plasma axis using a precise XYZ-holder with a rotating table. Both of the delay and gate times were adjusted to fixed levels of $1 \mu \mathrm{s}$ and $3 \mu \mathrm{s}$, respectively. The delay and gate times are chosen after many trails until we get the strongest observed line emission from the plasma with the minimum of background continuum radiation resulted from free-free and free-bound transitions. The background stray lights during the experiment were measured and subtracted and with the help of ICCD- KestrelSpec ${ }^{\circledR}$ software. The noise level resulted from the detection electronics quantum noise was measured across the whole wavelength region $(250-850 \mathrm{~nm})$ and was found as $20 \pm 7$ counts. The incident laser energy at each laser shot was measured utilizing a quartz beam splitter and the reflected part (4\%) is incident in an absolutely calibrated power-meter (Ophier model-1z02165). The laser energy was tuned in the range of laser energies using a set of calibrated neutral density filters. The data presented in this article are taken as the average over three consecutive shots onto fresh targets, and the date is presented together with standard deviations about means and plotted as error bars associated with the measurement points.

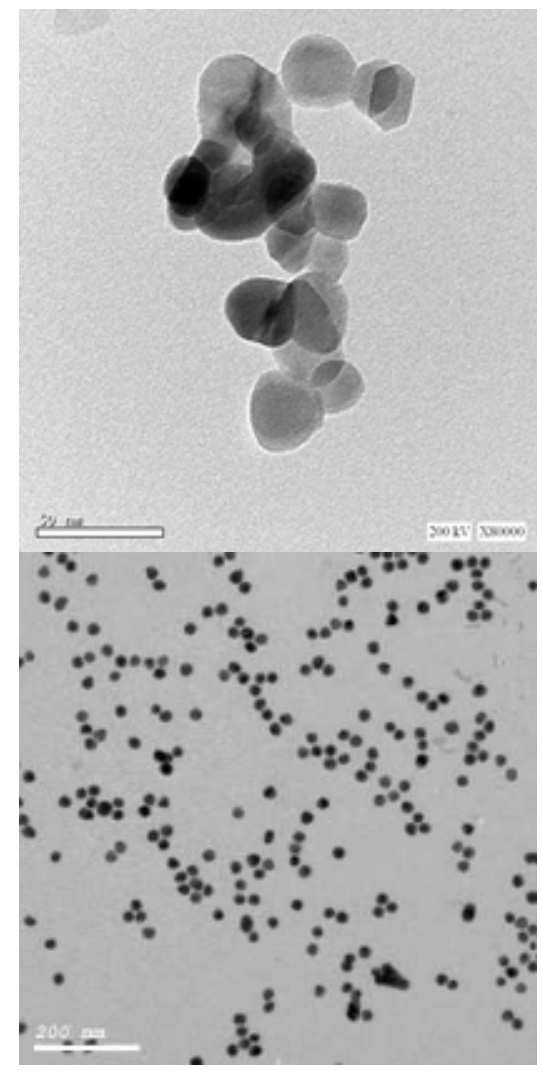

Fig. 2: Transmission electron microscope (TEM) of (a) titaniumnanoparticles, (b) gold-nanoparticles

The gold nanomaterial solution that purchased from "Nanotech CO" -prepared by chemical reduction method with a concentration of $(200 \mu \mathrm{g} / \mathrm{mL})$ in water- was deposited on the titanium metallic surface. A standard micro-pipette is used to drop $10 \mu \mathrm{L}$ on the surface and then the solution was evaporated to leave the gold NPs plated on the titanium surface. Transmission Electron Microscope (TEM) of titanium nanomaterial powder was characterized with the aid of the "Egyptian Petroleum Research Institute (EPRI)" as shown in Fig. 2(a).The nanoparticles were found to have a spherical shape with particle diameter (particle size) D 30 nm with a variance of $10 \mathrm{~nm}$. On the other side, the gold nanoparticles have spherical shapes with a diameter ( $\mathrm{D} \sim 20 \mathrm{~nm}$ ) measured using TEM as shown in Fig. 2(b), and no distortions were observed. The bulk titanium standard sample TI-M-04-SAMP was also purchased from American elements ${ }^{\circledR}$ with a purity of 99.9 $\%$ on the form of a flat surface disk of diameter $3 \mathrm{~cm}$. The 
plasma ignition was monitored using the lines at 453.32, $498.17 \mathrm{~nm}$ emitted by the neutral titanium as the parameter of plasma creation.

\section{RESULTS AND DISCUSSION}

A parametric study will be performed to illustrate the relation between the signal-to-noise ratio and the laser fluence to get the plasma ignition threshold (laser flounce threshold).

A. Enhanced emission from pure titanium nanomaterialcover bulk one at $1064 \mathrm{~nm}$ laser wavelength

The two Ti I-spectral lines at wavelengths of 453.32 and $498.17 \mathrm{~nm}$ are selected for performing analysisbecause they are not resonance lines i.e. they are not affected by self-absorption.

The light emitted from plasma from $250-850 \mathrm{~nm}$ is shown in Fig. 3. The blue curve represents the spectrum radiated from the bulk target surface and the emission from the nanomaterial target is in red. There is a noticeable increase in the intensity of the spectral lines radiated from the Nano-titanium target.

The spectral line at $453.2 \mathrm{~nm}$ was picked out fine inspection. The line enhancement is shown in Fig. (5.4) as recorded from both bulk and Nano-titanium target (solid lines) and one consists of nanoparticles (dashed lines) at different values of laser flounce1.9 J/cm2 and $3 \mathrm{~J} / \mathrm{cm} 2$, respectively. The gain is found to be nine at the lower laser fluence value and three at the higher one.

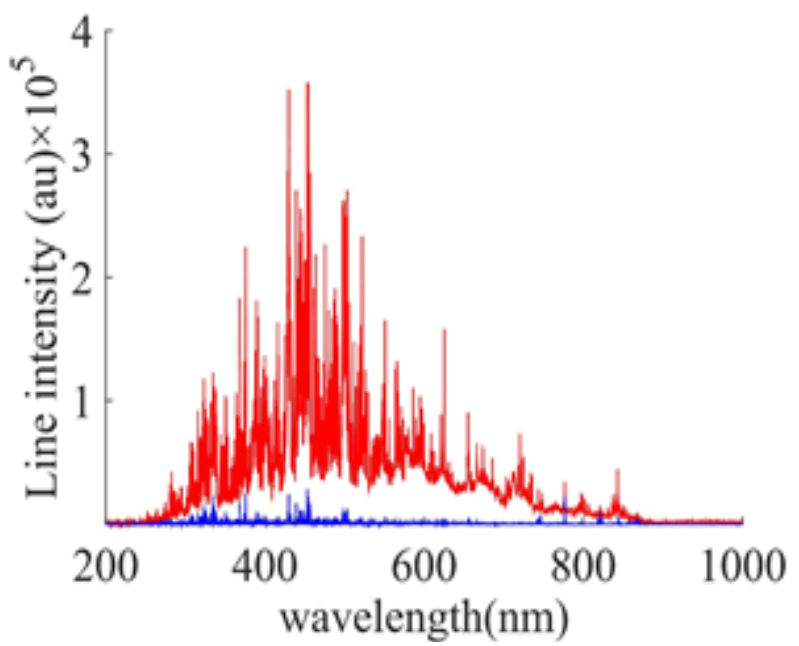

Fig. 3: Plasma emitted spectrum from bulk titanium (blue lines) and nano-titanium (red lines).
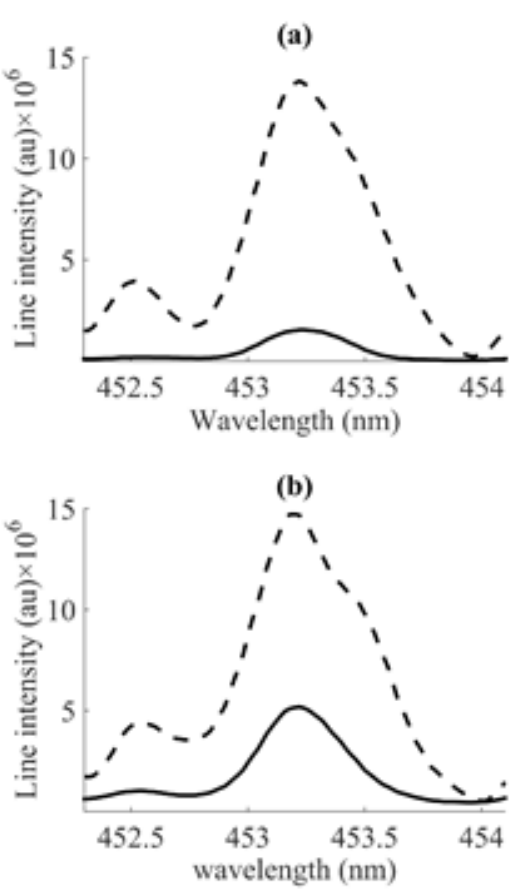

Fig. 4: Line intensity at $453.32 \mathrm{~nm}$ of both from bulk (solid line) and nanomaterial (dash line) (a) $\varphi=1.9 \mathrm{~J} / \mathrm{cm} 2$, (b) $\varphi=3 \mathrm{~J} / \mathrm{cm} 2$.

At another spectral line at wavelength of $498.17 \mathrm{~nm}$ is shown in Fig. 5. This line intensity was noticed to be increased by factor sixteen at laser fluence equal $1.9 \mathrm{~J} / \mathrm{cm}^{2}$ and this enhancement factor lowered to $5.5 \mathrm{~J} / \mathrm{cm}^{2}$ at laser fluence $3 \mathrm{~J} / \mathrm{cm}^{2}$.
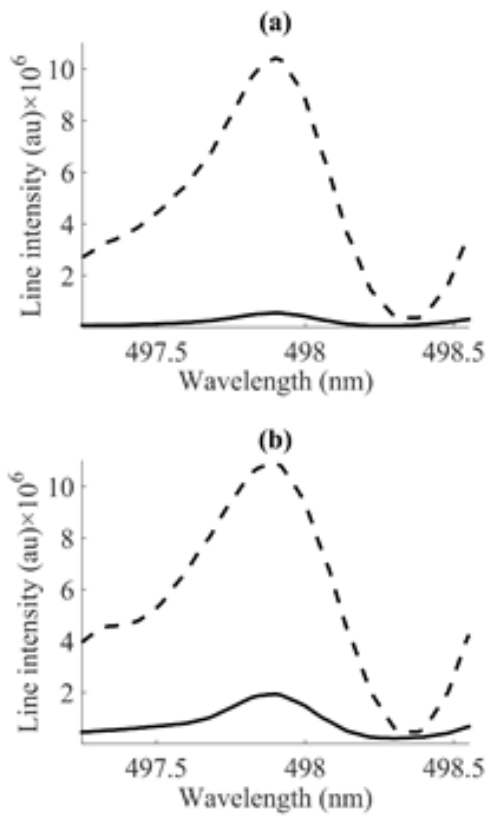

Fig. 5: Line intensity at $498.17 \mathrm{~nm}$ of both from bulk (solid line) and nanomaterial (dash line) (a) $\varphi=1.9 \mathrm{~J} / \mathrm{cm} 2$, (b) $\varphi=3 \mathrm{~J} / \mathrm{cm}^{2}$. 
Further, the laser flounce was varied at range tends from $1.7 \mathrm{~J} / \mathrm{cm} 2$ up to $6.4 \mathrm{~J} / \mathrm{cm} 2$ and the decrease of enhancement factor of the two analyzed spectral lines 453.32 and 498.17nm areshown in Fig. 6 (a) and (b), respectively.

One can notice the increase in the enhancement factor with the decrease of the laser fluence. The maximum enhancement factor of $453.32 \mathrm{~nm}$ spectral line was twelve at laser fluence $1.7 \mathrm{~J} / \mathrm{cm}^{2}$ and decreases exponentially till a value close to unity at laser fluence $6.4 \mathrm{~J} / \mathrm{cm} 2$. In the same way, the largest value of enhancement factor of the spectral line of wavelength $498.17 \mathrm{~nm}$ is twenty-two at the lowest laser fluence and also decreases exponentially till reaching unity at $6.4 \mathrm{~J} / \mathrm{cm}^{2}$.

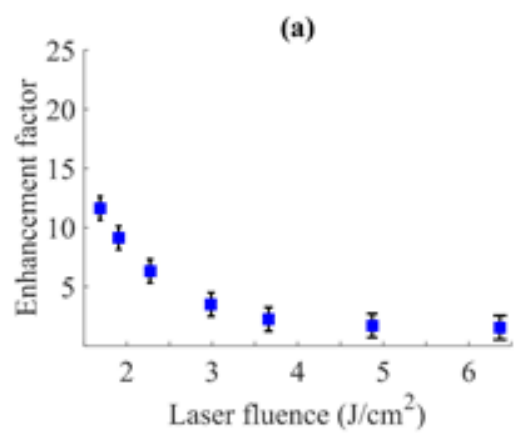

(b)

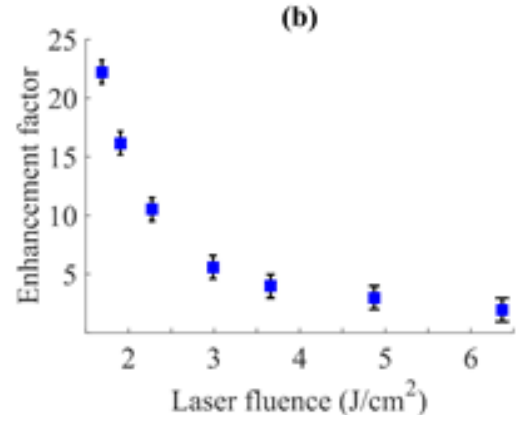

Fig. 6. Variation of enhancement factor at a different laser fluence of Ti I lines (a) $453.32 \mathrm{~nm}$, (b) $498.17 \mathrm{~nm}$.

As discussed in refs. $\left.{ }^{[25,26}\right]$, the plasma fluence threshold playing an important role in plasma creation in LIBS. In this discussion we shall recommend the thermodynamical point of view, that is the threshold flounce of plasma ignition can be calculated ${ }^{[14]}$ using the semi-empirical relation (1) for bulk-titanium and equation(3) for Nanotitanium by substitution with the titanium properties.

$$
\varphi_{t h}=\left(\rho L_{v}+2.235 \times 10^{15} \frac{E_{i}}{\lambda^{2}}\right) l_{T}
$$

Where, $\rho$ material density, latent heat of vaporizationL_v, ionization energy $E_{i}$, and exciting laser wavelength $\lambda$.

$$
\text { where, } \quad l_{T}=\sqrt{K_{T} \tau_{l} / \rho C_{p}}
$$

Where, $l_{T}$ is the thermal diffusion length in the solid material (with thermal conductivity coefficient $K_{T}$, heat capacity $C_{p}$ ) during the laser irradiation time $\tau_{l}$.

$$
\varphi_{t h}=\left(\rho L_{v}+2.235 \times 10^{15} \frac{E_{i}}{\lambda^{2}}\right) D
$$

At the beginning of fluence threshold calculation, it is essential to calculate thermal conduction length for titanium by substitution in (2) knowing that the laser pulse duration is $5 \pm 1 \mathrm{~ns}$ it is found that the thermal conduction length (IT) equals $215 \mathrm{~nm}$. The relation between signal-tonoise ratio and laser fluence was shown in Fig. 7 is used to illustrate the drop found in fluence threshold by using nanomaterial targets from bulk titanium.

(a)

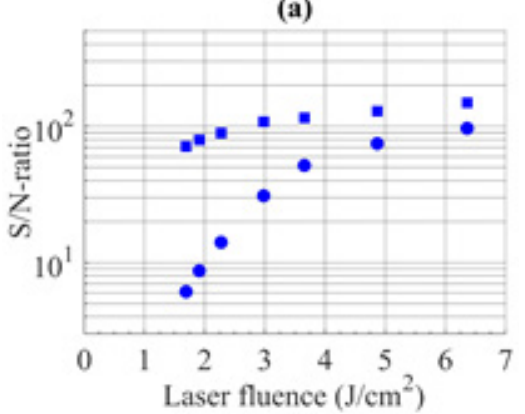

(b)

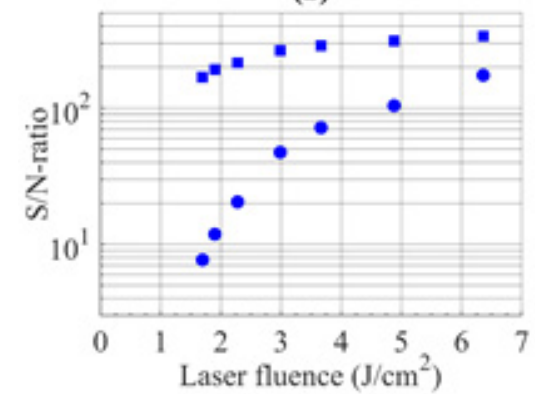

Fig. 7: The signal-to-noise ratio of Ti I lines at laser wavelength $1064 \mathrm{~nm}$ for (a) $453.32 \mathrm{~nm}$, (b) $498.17 \mathrm{~nm}$ versus laser fluence of both bulk titanium (circles) and Nano- titanium (squares).

\section{B. PLASMA RADIATION ENHANCEMENT USING GOLD NANOPARTICLES AT 1064 NM LASER WAVELENGTH}

A general display of the resulted emission from titanium target covered with thin layer of the $20 \mathrm{~nm}$ gold nanoparticles sheet is shown in Fig. 8 in red color; in comparison to emission from pure clean titanium target (blue). 


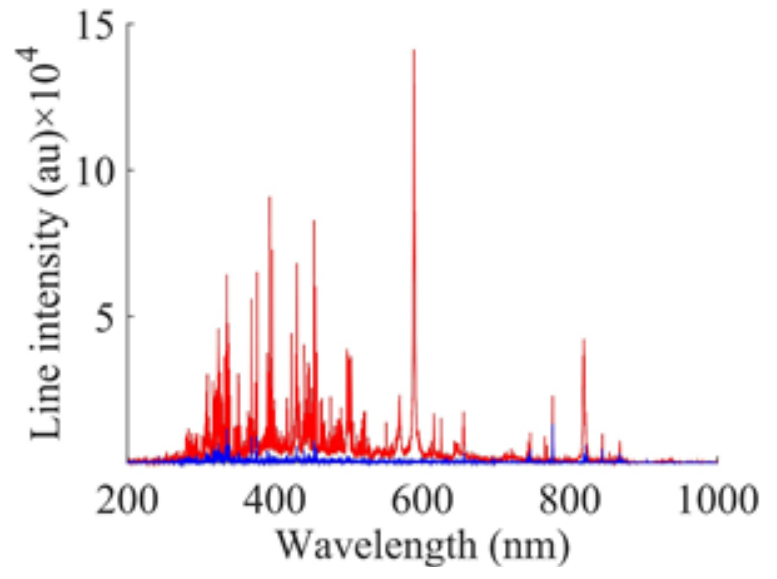

Fig. 8: Plasma emitted spectrum from bulk titanium (blue lines) and Nano-gold coated titanium (red lines) at laser wavelength $1064 \mathrm{~nm}$.

On the other hand, we have realized the effect of gold nanoparticles on enhancing the small signals from titanium. Quantitatively, Fig. 9 and Fig. 10 show the spectral line intensity at the two prominent Ti I-lines at wavelengths $453.32,498.17 \mathrm{~nm}$, respectively, as recorded from both of the pure titanium target (solid lines) and the one coated with gold nanoparticles (dashed lines). An apparent increase (enhancement) is obvious just after the addition of the Nano-gold thin sheet. It is worth noting that, the spectral radiance enhancement factor has greater value at the smaller laser fluence of $5.24 \mathrm{~J} / \mathrm{cm}^{2}$ and vice versa at the larger one with a value of $9.7 \mathrm{~J} / \mathrm{cm}^{2}$.

Nevertheless, the variation of the enhancement factor of the two spectral lines at 453.32 and $498.17 \mathrm{~nm}$ with laser fluence is presented in Fig. 11 in the range from $20 \mathrm{~J} / \mathrm{cm} 2$ down to $4 \mathrm{~J} / \mathrm{cm}^{2}$. An obvious decrease in the signal enhancement factor with laser fluence until reaching a value close to one at the highest fluence of $12.9 \mathrm{~J} / \mathrm{cm} 2$ is shown in Fig. $11(a, b)$ at the Ti I wavelengths of 453.4 and $498.17 \mathrm{~nm}$, respectively. This enhancement of Ti I-lines attests that the limit of detection of the LIBS technique is improved by the same ratio of recorded enhancement factors up to a factor of 25 at the lower irradiance levels.

In orderto explore the reason lying behind this changes in the enhancement factor with increasing laser fluence, a plot of the signal height arises from both of pure bulk Ti (lower points) was brought into comparison with the Nanogold-covered $\mathrm{Ti}$ (upper points) is presented at Fig. 12 $(a, b)$. The laser energy was detuned using a set of calibrated neutral density filters and the signals heights were recorded. The backward extrapolation leads to determine the laser threshold fluence from both targets.

In fact, we have assumed that there is a linear regression between line intensity to the background in the lower laser irradiance because of the absence of the effect of self-absorption. One can see that the theoretical value of the laser fluence threshold in case of bulk-based titanium is calculated using equations $(1,2)$ and found to be $0.91 \mathrm{~J} / \mathrm{cm}^{2}$ which is very close to the experimental result that is equal $0.9 \pm 0.1 \mathrm{~J} / \mathrm{cm}^{2}$ and also experimentally, the laser fluence threshold is found to be $0.025 \pm 0.005 \mathrm{~J} / \mathrm{cm}^{2}$ in gold-deposited titanium as shown inFig. 12 for both spectral lines at 453.32 and $498 \mathrm{~nm}$.

It is worth noting that, these measured differences in plasma ignition threshold from bulk to Nano-deposited gold value is lower by a factor come on 40 than the bulky one ${ }^{[25,26]}$

We have tried to predict the value of the ignition threshold after the addition of the nano-gold layer $\left(0.025 \mathrm{~J} / \mathrm{cm}^{2}\right)$ by assuming that the thermal conduction length of the Nano-gold is reduced to the limit of the nanoparticle size diameter ${ }^{[14]}$ but the result leads to the threshold of value $0.066 \mathrm{~J} / \mathrm{cm} 2$ which still far from the experimentally measured one by a factor of three.

It is worth noting that, these measured differences in plasma ignition threshold from bulk to nano-deposited gold value is lower by a factor come on 40 than the bulky one and is still far from the ratio of the thermal conduction length $(216 \mathrm{~nm})$ to the diameter of the deposited nanomaterial $(20 \mathrm{~nm})$. This requires further theoretical modeling in the prior time.

One possible explanation based on the assumption that the gold-nanoparticles have large absorption to the incident laser light at a resonance wavelength of $520 \mathrm{~nm}$ by the process of surface plasmon resonance $(\mathrm{SPR})^{[22]}$, and hence, these particles are fragmented into much smaller ones carrying a sufficient energy to break the interatomic forces of the titanium solid and leading to much lower vaporization, while an effort should be carried in order to put a satisfactory physical explanation at the IR laser radiation at $1064 \mathrm{~nm}$ which far from the plasmon resonance .

(a)
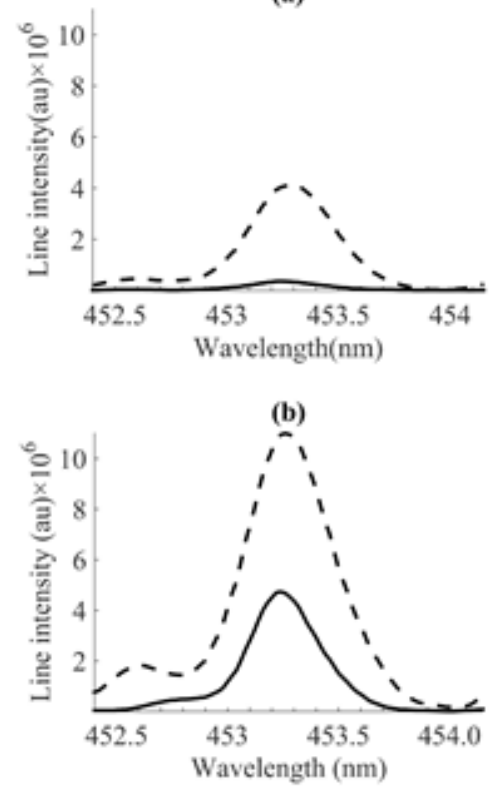

Fig. 9: Spectral line intensity of $453.32 \mathrm{~nm}$ from both of bulk (solid line) and nano-coated $\mathrm{Ti}$ (a) $5.25 \mathrm{~J} / \mathrm{cm} 2$, (b) $9.7 \mathrm{~J} / \mathrm{cm}^{2}$. 
(a)

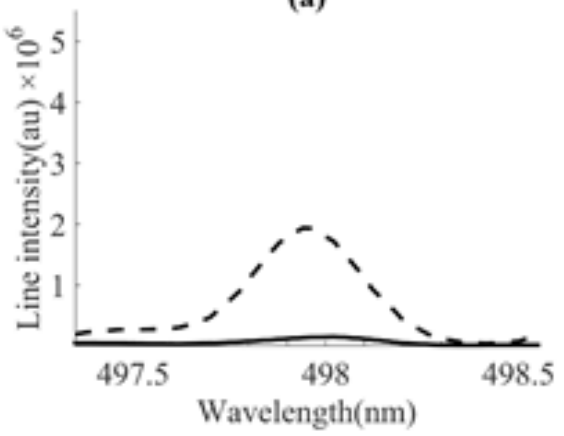

(b)

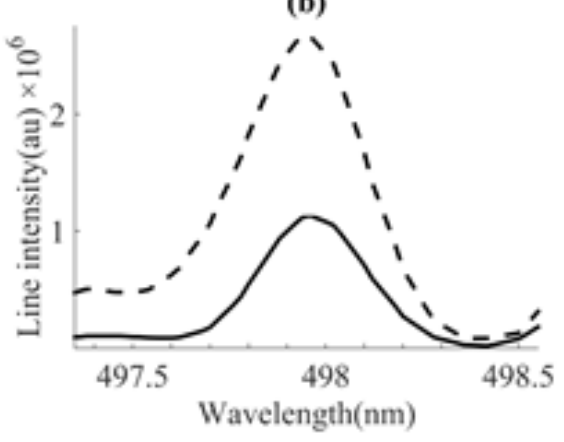

Fig. 10: Spectral line intensity of $498.17 \mathrm{~nm}$ from both of bulk (solid line) and nano-coated $\mathrm{Ti}$ (a) $5.25 \mathrm{~J} / \mathrm{cm} 2$, (b) $9.7 \mathrm{~J} / \mathrm{cm} 2$.
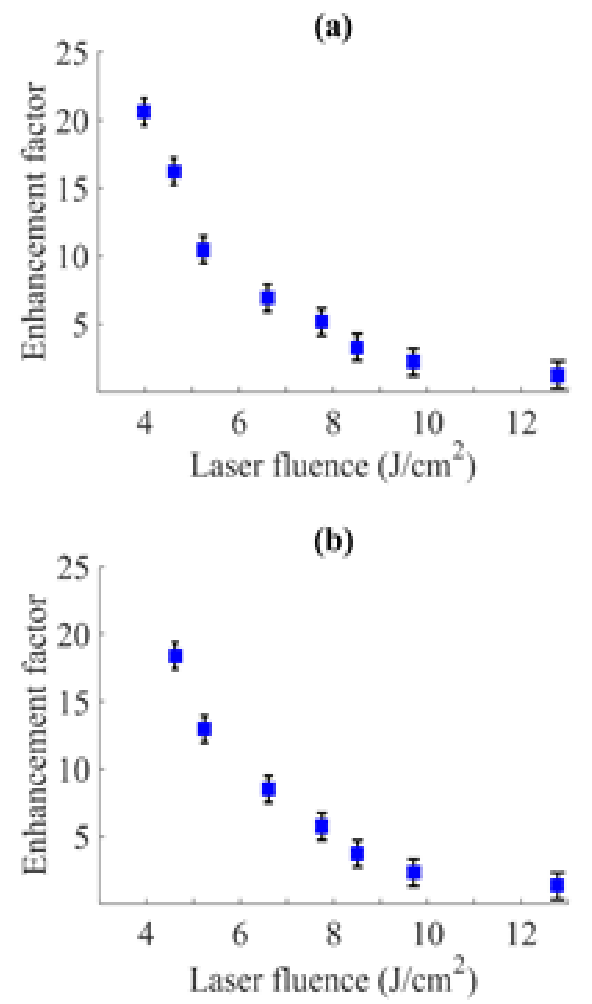

Fig. 11: Variation of enhancement factor at different laser fluence at different Ti I lines (a) $453.32 \mathrm{~nm}$, (b) $498.17 \mathrm{~nm}$. (a)

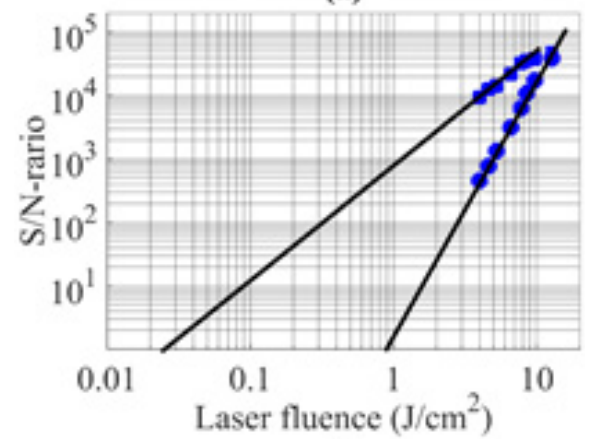

(b)

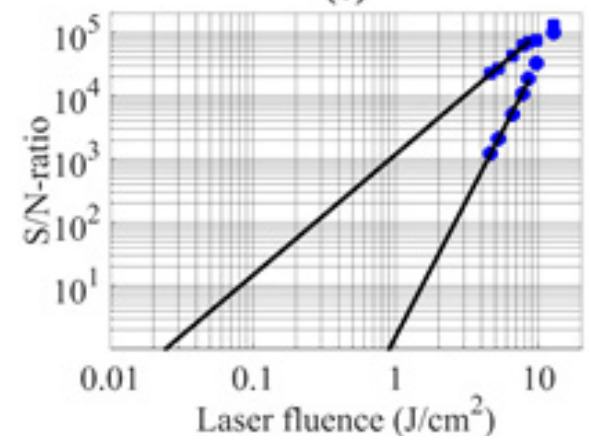

Fig. 12: The signal-to-noise ratio of Ti I lines at (a) $453.32 \mathrm{~nm}$, (b) $498.17 \mathrm{~nm}$ versus laser fluence of both bulk titanium (circles) and Nano-gold enhanced titanium (squares).

\section{CONCLUSIONS}

The enhancement of the radiation intensity using nanomaterial was demonstrated for two separated targets bulkand Nano-made titanium and extracted data ensure the enhancement of the signal intensity. The enhancement factor was found decreases by increasing laserflounce. The experimental study ensured that there is a decrease in the fluence thresholds in case of using titanium nanoparticles. The second methoddemonstrates enhancement of the emitted signal from plasma by depositing a thin layer of gold nanoparticles on a titanium metallic surface. For Nano-gold coated titanium, the laser fluence threshold was found experimentally to be lower than pure bulk titanium which suggests further studies.

\section{ACKNOWLEDGMENT}

The authors acknowledge the use of the facilities at the University of Cairo, Lab., of Lasers and New materials under the supervision of Prof. Th. M. El Sherbini, Dr. A. M. Aboulfotouh, as well as continuous and generous support from all the members of Laser Technology center, Military Technical College.

\section{REFERENCES}

[1] D. W. Hahn and N. Omenetto, "Laser-induced breakdown spectroscopy (LIBS), part II: review of instrumental and methodological 
approaches to material analysis and applications to different fields," Applied spectroscopy, vol. 66, no. 4, pp. 347-419, 2012.

[2] H.-J. Kunze, Introduction to plasma spectroscopy. Springer Science and Business Media, 2009.

[3] D. A. Cremers, F. Y. Yueh, J. P. Singh, and H. Zhang, Laser-Induced Breakdown Spectroscopy, Elemental Analysis. Wiley Online Library, 2006.

[4] R. S. Harmon, R. E. Russo, and R. R. Hark, "Applications of laserinduced breakdown spectroscopy for geochemical and environmental analysis: A comprehensive review," Spectrochimica Acta Part B: Atomic Spectroscopy, vol. 87, pp. 11-26, 2013.

[5] D. Rusak, B. Castle, B. Smith, and J. Winefordner, "Fundamentals and applications of laser-induced breakdown spectroscopy," Critical Reviews in Analytical Chemistry, vol. 27, no. 4, pp. 257-290, 1997.

[6] O. Samek et al., "Application of laser-induced breakdown spectroscopy to in situ analysis of liquid samples," Optical Engineering, vol. 39, no. 8, pp. 2248-2263, 2000.

[7] A. C. Samuels, F. C. DeLucia, K. L. McNesby, and A. W. Miziolek, "Laser-induced breakdown spectroscopy of bacterial spores, molds, pollens, and protein: initial studies of discrimination potential," Applied optics, vol. 42, no. 30, pp. 6205-6209, 2003.

[8] R. Wiens et al., "Pre-flight calibration and initial data processing for the ChemCam laser-induced breakdown spectroscopy instrument on the Mars Science Laboratory rover," Spectrochimica Acta Part B: Atomic Spectroscopy, vol. 82, pp. 1-27, 2013.

[9] M. Z. Martin et al., "High resolution applications of laser-induced breakdown spectroscopy for environmental and forensic applications," Spectrochimica Acta Part B: Atomic Spectroscopy, vol. 62, no. 12, pp 1426-1432, 2007.

[10] R. Noll et al., "Laser-induced breakdown spectroscopy expands into industrial applications," Spectrochimica Acta Part B: Atomic Spectroscopy, vol. 93, pp. 41-51, 2014.

[11] A. Giakoumaki, K. Melessanaki, and D. Anglos, "Laser-induced breakdown spectroscopy (LIBS) in archaeological science-applications and prospects," Analytical and bioanalytical chemistry, vol. 387, no. 3, pp. 749-760, 2007.

[12] A. W. Miziolek, V. Palleschi, and I. Schechter, Laser induced breakdown spectroscopy. Cambridge University Press, 2006.

[13] W. T. Y. Mohamed, "Improved LIBS limit of detection of Be, Mg, $\mathrm{Si}, \mathrm{Mn}, \mathrm{Fe}$ and $\mathrm{Cu}$ in aluminum alloy samples using a portable Echelle spectrometer with ICCD camera," Optics and Laser Technology, vol. 40, no. 1 , pp. 30-38, 2008.

[14] A. M. EL Sherbini and C. G. Parigger, "Wavelength dependency and threshold measurements for nanoparticle-enhanced laser-induced breakdown spectroscopy," Spectrochimica Acta Part B: Atomic Spectroscopy, vol. 116, pp. 8-15, 2016.
[15] G. L. Long and J. D. Winefordner, "Limit of detection. A closer look at the IUPAC definition," Analytical Chemistry, vol. 55, no. 7, pp 712A-724A, 1983.

[16] V. Babushok, F. DeLucia, J. Gottfried, C. Munson, and A. Miziolek, "Double pulse laser ablation and plasma: Laser induced breakdown spectroscopy signal enhancement," Spectrochimica Acta Part B: Atomic Spectroscopy, vol. 61, no. 9, pp. 999-1014, 2006.

[17] R. Sanginés and H. Sobral, "Time resolved study of the emission enhancement mechanisms in orthogonal double-pulse laser-induced breakdown spectroscopy," Spectrochimica Acta Part B: Atomic Spectroscopy, vol. 88, pp. 150-155, 2013.

[18] S. M. Angel, D. N. Stratis, K. L. Eland, T. Lai, M. A. Berg, and D. M. Gold, "LIBS using dual-and ultra-short laser pulses," Fresenius' journal of analytical chemistry, vol. 369, no. 3, pp. 320-327, 2001.

[19] D. Du, X. Liu, G. Korn, J. Squier, and G. Mourou, "Laser-induced breakdown by impact ionization in $\mathrm{SiO} 2$ with pulse widths from $7 \mathrm{~ns}$ to 150 fs," Applied physics letters, vol. 64, no. 23, pp. 3071-3073, 1994.

[20] J. Scaffidi, W. Pearman, M. Lawrence, J. C. Carter, B. W. Colston, and S. M. Angel, "Spatial and temporal dependence of interspark interactions in femtosecond-nanosecond dual-pulse laser-induced breakdown spectroscopy," Applied optics, vol. 43, no. 27, pp. 5243-5250, 2004.

[21] J. Scaffidi et al., "Dual-pulse laser-induced breakdown spectroscopy with combinations of femtosecond and nanosecond laser pulses," Applied optics, vol. 42, no. 30, pp. 6099-6106, 2003.

[22] A. De Giacomo, R. Gaudiuso, C. Koral, M. Dell'Aglio, and O. De Pascale, "Nanoparticle-enhanced laser-induced breakdown spectroscopy of metallic samples," Analytical chemistry, vol. 85, no. 21, pp. 10180 10187, 2013.

[23] A. De Giacomo, R. Gaudiuso, C. Koral, M. Dell'Aglio, and O. De Pascale, "Nanoparticle Enhanced Laser Induced Breakdown Spectroscopy: Effect of nanoparticles deposited on sample surface on laser ablation and plasma emission," Spectrochimica Acta Part B: Atomic Spectroscopy, vol. 98, pp. 19-27, 2014.

[24] A. M. El Sherbini, A.-N. M. Aboulfotouh, F. F. Rashid, S. H. Allam, A. El Dakrouri, and T. M. El Sherbini, "Observed enhancement in LIBS signals from nano vs. bulk $\mathrm{ZnO}$ targets: comparative study of plasma parameters," World Journal of Nano Science and Engineering, vol. 2, no. 04, p. 181, 2012.

[25] L. Cabalin and J. Laserna, "Experimental determination of laser induced breakdown thresholds of metals under nanosecond Q-switched laser operation," Spectrochimica Acta Part B: Atomic Spectroscopy, vol. 53, no. 5, pp. 723-730, 1998

[26] B. N. Chichkov, C. Momma, S. Nolte, F. Von Alvensleben, and A. Tünnermann, "Femtosecond, picosecond and nanosecond laser ablation of solids," Applied Physics A, vol. 63, no. 2, pp. 109-115, 1996. 